\title{
Robustness Analysis of Model Parameters for Sediment Transport Equation Development
}

\author{
Nadiatul Adilah Ahmad Abdul Ghani ${ }^{1}$, Duratul Ain Tholibon ${ }^{2}$ and Junaidah Ariffin $3^{*}$ \\ ${ }^{I}$ Faculty of Civil Engineering \& Earth Resources, Universiti Malaysia Pahang, \\ Lebuhraya Tun Razak, 26300 Gambang, Pahang, Malaysia \\ ${ }^{2}$ Faculty of Civil Engineering, Universiti Teknologi Mara (Jengka), \\ 26400 Jengka, Pahang, Malaysia \\ ${ }^{3}$ Faculty of Civil Engineering, Universiti Teknologi Mara (Shah Alam), \\ 40450 Shah Alam, Selangor, Malaysia
}

\begin{abstract}
Robustness analysis of model parameters for sediment transport equation development is carried out using 256 hydraulics and sediment data from twelve Malaysian rivers. The model parameters used in the analyses include parameters in equations by Ackers-White, Brownlie, EngelundHansen, Graf, Molinas-Wu, Karim-Kennedy, Yang, Ariffin and Sinnakaudan. Seven parameters in five parameter classes were initially tested. Robustness of the model parameters was measured on the statistical relations through Evolutionary Polynomial Regression (EPR) technique and further examined using the discrepancy ratio of the predicted versus the measured values. Results from analyses suggest $\frac{U^{*}}{V}$ (ratio of shear velocity to flow velocity) and $\frac{R}{d_{50}}$ (ratio of hydraulic radius to mean sediment diameter) to be the most significant and influential parameters for the development of sediment transport equation.
\end{abstract}

Keywords: reliability assessment; sediment transport

\section{INTRODUCTION}

Sediment transport is important in the fields of sedimentary geology, geomorphology, civil and environmental engineering. Knowledge of sediment transport is essential to help solve problems of deposition in navigation canals obstructing water traffic. Deposition problems in lakes are causing overflow with even brief storm events which affect the property at the perimeter of the lake. Local scouring around hydraulic structures and bridge piers, as well as bed and bank instability, is resulting from head-cutting due to sand and gravel mining activities. In sediment transport analysis, two types of loads are considered in the calculation, and they are the suspended load and bed load. Suspended load are loads that move in suspension with a diameter size of $0.0625 \mathrm{~mm}$ and larger. Usually, sand size of $2 \mathrm{~mm}$ diameter and smaller would remain buoyant and easily lifted depending on the hydraulics force of water. While bed loads are the bigger fractions that move by the traction force of the flow. Having to choose the most reliable model require a model assessment to be carried out. Assessment can be carried out using statistical relations and EPR technique of all model parameters of an equation.

$\mathrm{EPR}$ is a data-driven hybrid regression technique developed by Giustolisi and Savic (2006). EPR has been used successfully in solving several problems in civil engineering, e.g. (Ghorbani \& Hasanzadehshooiili 2018; Doglioni \& Simeone 2017; Yin et al., 2016; Giustolisi et al., 2008; Savic et al., 2006). It constructs symbolic models by integrating the best features of numerical regression (Draper \& Smith, 1998), with genetic programming and symbolic regression (Koza, 1992).

This paper aims to establish the most significant and influential parameters for use in the development of 
sediment transport equation. Parameter test analyses are carried out using statistical analysis and EPR technique.

\section{SEDIMENT TRANSPORT EQUATIONS - EVALUATIONS AND PERFORMANCES}

Sediment transport equations were developed mainly from flume experiments of shallow flows with depths not exceeding 0.5m (Ackers \& White 1973; Yang 1973; Engelund \& Hansen 1967). The derived equations are only suitable for use in channels of uniform flow and crosssection. Some adjustment on the predicted values may be required if used on natural rivers.

Evaluations of established sediment transport equations for use in Malaysian rivers have been carried out in the past (Department of Drainage and Irrigation 2009; Chang et al., 2005). The studies have identified two equations, Yang and Engelund-Hansen of acceptable performance. Yang derived his equation using data from the Yellow river consisting primarily of fine silts and clays. The suitability of Yang equation to predict sediment load in Malaysian rivers can be attributed to the similarity in sediment characteristics to China where most upland erosions originated from the loess region. The local researchers, Saleh et al. (2017), Sinnakaudan et al. (2006) and Ariffin (2004) have made efforts to develop sediment transport equations that are exclusive for Malaysian rivers.

Table 1 illustrates the performance of nine sediment transport equations on Malaysian rivers, namely of Sungai Perak, Sungai Kemaman, Sungai Pergau and Sungai Kurau and the corresponding model parameters modified after Saleh et al. (2017). The nine equations used in analyses are Molinas and Wu (2001), Karim (1998), Yang (1973), Graf (1971) and Engelund and Hansen (1967). Table 2 shows the data range for $d_{50}$ used in the analyses (Saleh 2016).

Table 1. Performance of nine sediment transport equations on Malaysian rivers and the corresponding model parameters (modified after Saleh et al., 2017)

\begin{tabular}{|c|c|c|c|}
\hline Equation & Model parameters & $\begin{array}{c}\text { Data within (0.5- } \\
\text { 2.0)DR }\end{array}$ & Percentage \\
\hline Ackers-White (1973) & $\frac{V}{u_{*}}, \frac{F_{g r}}{A}, d_{35}, d_{50}, \frac{\Delta g}{v^{2}}$ & 14 & 40 \\
\hline Ariffin (2004) & $R / d_{50}, U^{*} / W_{s}, U^{*} / V, V^{2} / g y_{o}$ & 29 & 83 \\
\hline $\begin{array}{l}\text { Engelund and } \\
\text { Hansen (1967) }\end{array}$ & $\gamma_{s}, V^{2}, \sqrt{d_{50} / g\left(\gamma_{s} / \gamma_{w}\right)}, \sqrt[1.5]{\tau /\left(\gamma_{s}-\gamma_{w}\right) d_{50}}$ & 6 & 17 \\
\hline Graf (1971) & $\left(S_{s}-1\right) d_{50} / R S_{o}, C_{v} V R / \sqrt{g\left(S_{s}-1\right) d_{50}^{3}}$ & 4 & 11 \\
\hline $\begin{array}{l}\text { Molinas and Wu } \\
\text { (2001) }\end{array}$ & $V, g, w_{s}, d_{50}, h$ & 22 & 63 \\
\hline Karim (1998) & $\frac{u_{*}}{w_{s}}, V, g, d_{50}$ & 20 & 57 \\
\hline $\begin{array}{l}\text { Sinnakaudanet al. } \\
\text { (2006) }\end{array}$ & $V S_{o} / \omega_{s}, R / d_{50}, \sqrt{g\left(S_{s}-1\right) d_{50}^{3}} / V R$ & 24 & 69 \\
\hline Yang (1973) & $, \frac{u_{*}}{w_{S}}, \frac{V S}{W_{s}}, R_{e}, \frac{w_{s} d_{50}}{v}$ & 9 & 26 \\
\hline
\end{tabular}


Table 2. Total bed material load and data range for $\mathrm{d}_{50}$ (Saleh 2016)

\begin{tabular}{|l|l|}
\hline \multicolumn{1}{|c|}{ Equation } & Data Range $(\mathrm{mm})$ \\
\hline Graf & $0.09<\mathrm{d}_{50}<2.78$ \\
\hline Engelund and Hansen (E-H) & $0.19<\mathrm{d}_{50}<0.93$ \\
\hline Yang & $0.137<\mathrm{d}_{50}<1.71$ \\
\hline Ackers and White & $0.04<\mathrm{d}_{50}<4.94$ \\
\hline Ariffin & $0.37<\mathrm{d}_{50}<4.00$ \\
\hline Sinnakaudanetal. & $0.3711<\mathrm{d}_{50}<4.00$ \\
\hline
\end{tabular}

\section{DATA SELECTION}

Data used in this study comprised of data from the works of Saleh et al. (2017), Department of Irrigation and Drainage (2013), Ibrahim (2012) and Ariffin (2004). There are 256 hydraulics, and sediment data measured from twelve rivers in Malaysia and the data range is given in Table 3.

\section{A. Performance of Sediment Transport Equations on Twelve Rivers by Various Investigators}

Table 4 shows the range of hydraulics and sediment data used in the analyses that include width, velocity and median size of sediment load. Table 5 illustrates the performance of selected equations on Malaysian river data.
Table 3. Data range used in analyses

\begin{tabular}{|l|c|}
\hline \multicolumn{1}{|c|}{ Parameter } & Range \\
\hline Total sediment load, $T_{j}(\mathrm{~kg} / \mathrm{s})$ & $0.0333-119.601$ \\
\hline Flow, $Q\left(\mathrm{~m}^{3} / \mathrm{s}\right)$ & $0.737-87.792$ \\
\hline Velocity, $V(\mathrm{~m} / \mathrm{s})$ & $0.194-1.422$ \\
\hline Depth of water, $y_{0}(\mathrm{~m})$ & $0.22-3.23$ \\
\hline Particle mean size, $d_{50}(\mathrm{~mm})$ & $0.37-4.9109$ \\
\hline Water surface slope, $S_{\mathrm{o}}$ & $0.0003-0.0167$ \\
\hline Fall velocity, $W_{\mathrm{s}}(\mathrm{m} / \mathrm{s})$ & $0.043-21.157$ \\
\hline Hydraulic radius, $R(\mathrm{~m})$ & $0.21-2.66$ \\
\hline
\end{tabular}

Table 4. Range of hydraulics and sediment data used in analyses

\begin{tabular}{|c|l|c|c|c|c|}
\hline No & \multicolumn{1}{|c|}{ River } & $\begin{array}{c}\text { Total no } \\
\text { of data }\end{array}$ & Width $(\mathrm{m})$ & Velocity (m/s) & $\begin{array}{c}\text { Median sediment size, } \mathrm{d}_{50} \\
(\mathrm{~mm})\end{array}$ \\
\hline 1 & Raia & 41 & 17.325 .6 & $0.478-0.76$ & $0.5-1.6$ \\
\hline 2 & Kulim & 16 & $14-18$ & $0.303-0.872$ & $0.4-1$. \\
\hline 3 & Kinta & 20 & $24.6-28$ & $0.42-0.651$ & $2.8-3.0$ \\
\hline 4 & Kerayong & 24 & 18 & $0.218-0.586$ & $0.526-2.471$ \\
\hline 5 & Bernam & 36 & $12-20$ & $0.266-0.868$ & $0.879-2.288$ \\
\hline 6 & Semenyih & 50 & $13.5-15$ & $0.447-0.852$ & $0.85-1.1$ \\
\hline 7 & Kampar & 21 & $20.2-21$ & $0.592-0.71$ & $0.502-1.758$ \\
\hline 8 & Langat & 23 & $17-30$ & $0.536-1.422$ & $1.01-2.62$ \\
\hline 9 & Lui & 92 & $15-17$ & $0.194-1.029$ & $0.715-1.27$ \\
\hline 10 & Jeli & 3 & $31-46$ & $0.43-0.69$ & $0.85-3.1$ \\
\hline 11 & Kurau & 56 & $18-20.3$ & $0.461-1.26$ & \\
\hline 12 & Pari & & & & $0.45-0.636$ \\
\hline
\end{tabular}




\section{B. Robustness Measurement}

The robustness measurement of all model parameters based on five parameter classes was derived from studies carried out by Ariffin (2017; 2004), Azamathulla et al. (2010), Sulaiman (2009), Sinnakaudan et al. (2006), and Chang et al. (2005). The variables are, relative roughness on the bed $\left(R / d_{50}\right)$ in flow resistance parameter class, stream-width ratio $\left(B / y_{0}\right)$ in conveyance and shape class which are shear velocity ratio to fall velocity $\left(U^{*} / \omega_{s}\right)$ and fall velocity to shear velocity $\left(\omega_{\mathrm{s}} / U^{*}\right)$, in sediment properties class is ratio of shear stress to average velocity $\left(U^{*} / V\right)$ and dimensionless unit stream power $\left(V S_{o} / \omega_{s}\right)$ in mobility class and the last variable is velocity head $\left(v^{2} / 2 g\right)$. The output variable selected for this model is concentration by volume or volumetric concentration (ratio of total sediment transport rate to flow rate) $(Q t / Q)$.

Data were randomly divided into two sets: a training set for model calibration and an independent validation set for model verification. In dividing the data into their sets, the training and testing sets were selected to be statistically consistent; thus, represent the same statistical population, as recommended by Shahin et al. (2004). In total, 174 data cases (68\%) of the 256 data cases are used for training and balance 82 data cases (32\%) for use for validation. The statistical analysis showing measures of central tendency (mean values) and variability (standard deviation, minimum and maximum values) is given in Table 6. Table 7 lists the model parameters used in the robustness analysis.

Table 5. Performance of selected equations on Malaysian river data

\begin{tabular}{|c|c|c|c|c|c|c|c|}
\hline \multirow[b]{2}{*}{ No. } & \multirow[b]{2}{*}{ River } & \multirow{2}{*}{$\begin{array}{l}\text { Total No of } \\
\text { data }\end{array}$} & \multicolumn{5}{|c|}{ Percentage of data within DR of $0.5-2.0$} \\
\hline & & & $\begin{array}{c}\text { Engelund } \\
\text { and Hansen } \\
\text { (1967) }\end{array}$ & Graf (1971) & $\begin{array}{l}\text { Ariffin } \\
(2004)\end{array}$ & $\begin{array}{c}\text { Chang et al. } \\
\text { (2005) }\end{array}$ & $\begin{array}{c}\text { Sinnakaudan et al. } \\
\text { (2006) }\end{array}$ \\
\hline 1 & Raia & 41 & $\mathrm{O}$ & $\mathrm{O}$ & 61 & 63 & 66 \\
\hline 2 & Kulim & 16 & 0 & 56 & 88 & O & 75 \\
\hline 3 & Kinta & 20 & 20 & 30 & 45 & 0 & 30 \\
\hline 4 & Kerayong & 24 & 21 & 50 & 83 & 0 & 58 \\
\hline 5 & Bernam & 36 & 39 & 17 & 25 & 11 & 28 \\
\hline 6 & Semenyih & 50 & 30 & 8 & 56 & 30 & 68 \\
\hline 7 & Kampar & 21 & 38 & $\mathrm{O}$ & $\mathrm{O}$ & 28 & 48 \\
\hline 8 & Langat & 23 & 17 & 0 & 43 & 13 & 57 \\
\hline 9 & Lui & 92 & 14 & 2 & 46 & 22 & 63 \\
\hline 10 & Jeli & 3 & 33 & 67 & O & 0 & 33 \\
\hline 11 & Kurau & 8 & 0 & $\mathrm{O}$ & $\mathrm{O}$ & 38 & 88 \\
\hline 12 & Pari & 56 & 43 & 25 & 34 & 2 & 73 \\
\hline
\end{tabular}


Table 6. Measures of central tendency and variability

\begin{tabular}{|c|c|c|c|c|c|}
\hline \multirow[b]{2}{*}{ Model parameters } & \multicolumn{5}{|c|}{ Measures of central tendency and variability } \\
\hline & Mean & $\begin{array}{l}\text { Standard } \\
\text { Deviation }\end{array}$ & Minimum & Maximum & Range \\
\hline \multicolumn{6}{|l|}{ Total load, $(Q t / Q)$} \\
\hline Training set & 0.0003569 & 0.0001926 & 0.00010 & 0.00093 & 0.00 \\
\hline Testing set & 0.0003974 & 0.0002484 & 0.00009 & 0.00215 & 0.00 \\
\hline \multicolumn{6}{|c|}{ Ratio of stream radius to median diameter of bed material $\left(R / d_{5 o}\right)$} \\
\hline Training set & 428.19 & 216.87 & 106.90 & 1229.73 & 1122.84 \\
\hline Testing set & 642.33 & 445.03 & 213.35 & 2800.00 & 2586.65 \\
\hline \multicolumn{6}{|c|}{ Stream width ratio to water depth $\left(B / y_{o}\right)$} \\
\hline Training set & 32.29 & 13.72 & 10.86 & 87.76 & 76.91 \\
\hline Testing set & 33.08 & 14.89 & 9.29 & $74 \cdot 70$ & 65.41 \\
\hline \multicolumn{6}{|c|}{ Ratio of shear velocity to fall velocity $\left(U^{*} / \omega_{s}\right)$} \\
\hline Training set & 0.56 & 0.26 & 0.01 & 0.99 & 0.98 \\
\hline Testing set & 1.40 & 0.61 & 0.00 & 5.44 & $5 \cdot 43$ \\
\hline \multicolumn{6}{|c|}{ Ratio of fall velocity to shear velocity $\left(\omega_{s} / U^{*}\right)$} \\
\hline Training set & 4.95 & 15.85 & 1.01 & 153.47 & 152.45 \\
\hline Testing set & 3.41 & $24 \cdot 33$ & 0.18 & 225.08 & 224.89 \\
\hline \multicolumn{6}{|c|}{ Ratio of shear velocity to average velocity $\left(U^{*} / V\right)$} \\
\hline Training set & 0.18 & 0.08 & 0.07 & 0.47 & 0.39 \\
\hline Testing set & 0.26 & 0.11 & 0.10 & 0.73 & 0.63 \\
\hline \multicolumn{6}{|c|}{ Dimensionless unit stream power $\left(V S_{o} / \omega_{s}\right)$} \\
\hline Training set & 0.01 & 0.01 & 0.00 & 0.02 & 0.02 \\
\hline Testing set & 0.03 & 0.02 & 0.00 & 0.12 & 0.12 \\
\hline \multicolumn{6}{|c|}{ Velocity head $\left(V^{2} / 2 g\right)$} \\
\hline Training set & 0.07 & 0.03 & 0.01 & 0.16 & 0.14 \\
\hline Testing set & 0.08 & 0.03 & 0.01 & 0.14 & 0.13 \\
\hline
\end{tabular}


Table 7. Parameters used in robustness analysis

\begin{tabular}{|c|c|c|c|c|c|c|c|}
\hline Name & $\left(R / d_{50}\right)$ & $\left(B / y_{o}\right)$ & $\left(U^{*} / \omega_{s}\right)$ & $\left(\omega_{s} / U^{*}\right)$ & $\left(U^{*} / V\right)$ & $\left(V S_{o} / \omega_{s}\right)$ & $\left(V^{2} / 2 g\right)$ \\
\hline Model1 & $/$ & I & / & $/$ & I & $/$ & / \\
\hline Model2 & $/$ & I & $/$ & & I & & \\
\hline Model3 & I & I & 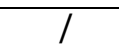 & 7 & 1 & & \\
\hline Model 4 & / & I & / & 1 & / & / & \\
\hline Model5 & / & I & 1 & I & & & \\
\hline Model6 & I & I & I & / & & I & \\
\hline Model7 & / & 7 & I & I & & I & I \\
\hline Model8 & / & I & 7 & & & & / \\
\hline Model9 & 1 & I & 1 & I & & & 1 \\
\hline Model10 & $/$ & I & $/$ & $/$ & I & & $/$ \\
\hline Model11 & $/$ & / & $/$ & & / & I & \\
\hline Model12 & $/$ & / & / & & & / & \\
\hline Model13 & / & / & / & & / & I & / \\
\hline
\end{tabular}

Selection of the model parameters was carried out by trialand-error approach in which a series of EPR models were trained using functions given in Table 8. A more detailed description of functions used for parameter selection can be found in the EPR Toolbox manual (Laucelli et al., 2009).

Table 8. Functions used for parameter selection

\begin{tabular}{|l|l|}
\hline Function & \multicolumn{1}{|c|}{ Expression Structure } \\
\hline $\mathrm{f}_{0}$ & $Y=\operatorname{sum}\left(a_{i}{ }^{*} X_{1}{ }^{*} X_{2}{ }^{*} f\left(X_{1}\right){ }^{*} f\left(X_{2}\right)\right)+a_{o}$ \\
\hline $\mathrm{f}_{1}$ & $Y=\operatorname{sum}\left(a_{i}{ }^{*} f\left(X_{1}{ }^{*} X_{2}\right)\right)+a_{o}$ \\
\hline $\mathrm{f}_{2}$ & $Y=\operatorname{sum}\left(a_{i}{ }^{*} X_{1}{ }^{*} X_{2}{ }^{*} f\left(X_{1}{ }^{*} X_{2}\right)\right)+a_{o}$ \\
\hline LS & Least square \\
\hline LSN & Non-negative least square \\
\hline
\end{tabular}

\section{Model Approximations}

Models are approximated using Least Square (LS) and NonNegative Least Square (LSN) methods. Accuracy of the models was measured using the discrepancy ratio of 0.5 2.o. Discrepancy ratio is the ratio of predicted to measured values. Performance of all models is shown in Table 9. Table 10 illustrates the best performing models of the model groups with the corresponding model exponential relations. The model parameters established from the robustness test are used as inputs in the EPR model. A total of 666 new models from 13 groups have been generated using 174 data in training set using the functions given in Table 8.

Table 9. Performance results of the EPR models during training

\begin{tabular}{|l|l|l|l|l|l|l|l|l|l|l|l|l|}
\hline Model group & \multicolumn{9}{|c|}{ Accuracy DR (0.5-2.0) (\%) } \\
\hline Model1 & $\mathbf{1}$ & $\mathbf{2}$ & $\mathbf{3}$ & $\mathbf{4}$ & $\mathbf{5}$ & $\mathbf{6}$ & 7 & $\mathbf{8}$ & $\mathbf{9}$ & $\mathbf{1 0}$ & $\mathbf{1 1}$ & $\mathbf{1 2}$ \\
\hline foLS & 70.11 & 70.11 & 70.11 & 70.11 & 74.71 & 77.59 & 75.86 & & & & \\
\hline f1LS & 69.94 & 69.94 & 69.94 & 69.94 & 74.56 & 75.72 & & & & & & \\
\hline f2LS & 71.10 & 71.10 & 58.38 & 71.10 & 71.10 & 65.32 & 63.01 & 63.01 & 75.72 & & & \\
\hline foLSN & 69.54 & 66.09 & 66.09 & 64.37 & 67.24 & 68.39 & 72.99 & 72.41 & 71.84 & 74.14 & 75.29 & \\
\hline f1LSN & 69.94 & 66.47 & 66.47 & 64.74 & 67.63 & 69.94 & 73.99 & 73.41 & 72.83 & 75.14 & 76.30 & \\
\hline f2LSN & 71.10 & 67.03 & 67.63 & 65.90 & 68.79 & 69.94 & 73.99 & 73.41 & 72.83 & 76.88 & 72.25 & 76.30 \\
\hline Model2 & $\mathbf{1}$ & $\mathbf{2}$ & $\mathbf{3}$ & $\mathbf{4}$ & $\mathbf{5}$ & $\mathbf{6}$ & 7 & $\mathbf{8}$ & $\mathbf{9}$ & $\mathbf{1 0}$ & $\mathbf{1 1}$ & $\mathbf{1 2}$ \\
\hline foLS & 69.54 & 69.54 & 69.54 & 69.54 & 69.54 & 69.54 & 69.54 & 69.54 & & & & \\
\hline
\end{tabular}




\begin{tabular}{|c|c|c|c|c|c|c|c|c|c|c|c|c|}
\hline f1LS & 69.94 & 69.94 & 69.94 & 69.94 & 69.94 & 69.94 & 69.94 & 69.94 & & & & \\
\hline f2LS & 71.10 & 87.86 & 87.86 & 78.61 & 78.61 & 100.00 & 100.00 & & & & & \\
\hline foLSN & 69.54 & 66.09 & 65.52 & 64.94 & 65.52 & 67.24 & 64.94 & 64.37 & 69.54 & & & \\
\hline f1LSN & 69.94 & 66.47 & 65.90 & 65.32 & 65.90 & 67.63 & $65 \cdot 32$ & 64.74 & 69.94 & & & \\
\hline f2LSN & 71.1 & 67.73 & 67.05 & 66.47 & 67.05 & 69.36 & 67.05 & & & & & \\
\hline Model3 & $\mathbf{1}$ & 2 & 3 & 4 & 5 & 6 & 7 & 8 & 9 & 10 & 11 & 12 \\
\hline foLS & 70.11 & 86.78 & 86.78 & 77.01 & 77.01 & 98.28 & 90.23 & & & & & \\
\hline f1LS & 69.94 & 86.71 & 86.71 & 76.88 & 76.88 & 98.27 & 90.17 & & & & & \\
\hline f2LS & 71.10 & 71.10 & 71.10 & 71.10 & 71.10 & 71.10 & 71.10 & 71.10 & 71.10 & & & \\
\hline foLSN & 69.54 & 66.09 & $65.5^{2}$ & 64.94 & 65.52 & 67.24 & $65.5^{2}$ & 64.94 & 70.11 & & & \\
\hline f1LSN & 69.94 & 66.47 & 65.90 & $65 \cdot 32$ & 65.90 & 69.36 & 66.47 & 65.90 & 71.86 & & & \\
\hline f2LSN & 71.10 & 67.63 & 67.05 & 66.47 & 67.05 & 69.36 & 67.05 & & & & & \\
\hline Model4 & $\mathbf{1}$ & 2 & 3 & 4 & 5 & 6 & 7 & 8 & 9 & 10 & 11 & 12 \\
\hline foLS & 70.11 & 70.11 & 70.11 & 70.11 & 70.11 & 70.11 & & & & & & \\
\hline f1LS & 69.94 & 69.94 & 69.94 & 69.94 & 69.94 & 69.94 & 69.94 & 65.90 & & & & \\
\hline f2LS & 71.10 & 71.10 & 76.88 & 76.88 & 76.30 & 76.30 & 75.14 & 72.83 & & & & \\
\hline foLSN & 69.54 & 66.09 & 64.94 & 64.37 & 66.09 & 63.79 & 72.99 & 73.56 & 74.71 & 71.26 & & \\
\hline f1LSN & 69.94 & 69.47 & $65 \cdot 32$ & 64.74 & 66.47 & $65 \cdot 32$ & 74.57 & 74.57 & 76.30 & 72.83 & & \\
\hline f2LSN & 71.10 & 67.63 & 66.47 & 65.90 & 67.63 & $65 \cdot 32$ & 74.57 & 75.57 & 76.30 & 72.83 & & \\
\hline Model5 & $\mathbf{1}$ & 2 & 3 & 4 & 5 & 6 & 7 & 8 & 9 & 10 & 11 & 12 \\
\hline foLS & 61.49 & 86.78 & 77.01 & 77.01 & & & & & & & & \\
\hline \multicolumn{13}{|l|}{ f1LS } \\
\hline \multicolumn{13}{|l|}{ f2LS } \\
\hline foLSN & 60.92 & 66.09 & 66.67 & 67.82 & 67.24 & 67.82 & 68.39 & 68.39 & & & & \\
\hline f1LSN & 61.27 & 66.47 & 67.05 & 68.21 & 67.63 & 69.36 & 69.36 & & & & & \\
\hline f2LSN & 67.63 & 68.21 & 68.21 & 68.79 & 69.36 & 69.36 & & & & & & \\
\hline Model6 & $\mathbf{1}$ & 2 & 3 & 4 & 5 & 6 & 7 & 8 & 9 & 10 & $\mathbf{1 1}$ & 12 \\
\hline foLS & 61.49 & 86.78 & 67.82 & 77.01 & 77.01 & 67.82 & & & & & & \\
\hline f1LS & 61.27 & 86.71 & 67.63 & 76.88 & 76.88 & 67.63 & & & & & & \\
\hline f2LS & 67.05 & 87.28 & 77.46 & 78.03 & 78.03 & & & & & & & \\
\hline foLSN & 60.92 & 66.09 & 66.09 & 65.52 & 70.11 & 67.82 & 68.39 & 68.39 & 68.39 & 68.39 & 68.39 & \\
\hline f1LSN & 61.27 & 66.47 & 66.47 & 65.90 & 70.52 & 69.36 & 69.36 & 69.36 & 69.36 & 69.36 & & \\
\hline f2LSN & 61.63 & 67.63 & 67.63 & 67.05 & 71.68 & 69.36 & 69.36 & 69.36 & & & & \\
\hline Model7 & 1 & 2 & 3 & 4 & 5 & 6 & 7 & 8 & 9 & 10 & 11 & 12 \\
\hline foLS & 67.24 & $\mathrm{O}$ & 68.39 & 64.37 & 70.11 & 70.11 & 70.11 & 70.11 & & & & \\
\hline f1LS & 66.47 & 0 & 67.03 & 63.58 & 69.36 & 69.36 & 69.36 & 69.36 & & & & \\
\hline f2LS & 67.63 & 67.63 & 69.36 & $65 \cdot 32$ & 67.63 & 67.63 & & & & & & \\
\hline foLSN & 66.67 & 66.09 & 66.67 & 72.99 & 72.99 & 69.54 & 66.67 & 71.26 & 70.69 & 68.39 & & \\
\hline f1LSN & 67.05 & 66.47 & 67.05 & 73.41 & 71.10 & 71.10 & 67.63 & 72.25 & 71.68 & 69.36 & & \\
\hline f2LSN & 67.63 & 67.63 & 68.21 & 67.05 & 69.05 & 69.36 & 69.36 & 76.30 & 73.41 & 73.99 & 74.57 & \\
\hline Model8 & $\mathbf{1}$ & 2 & 3 & 4 & 5 & 6 & 7 & 8 & 9 & 10 & 11 & 12 \\
\hline
\end{tabular}




\begin{tabular}{|c|c|c|c|c|c|c|c|c|c|c|c|c|}
\hline foLS & 67.24 & O & 68.39 & 64.37 & 70.11 & 70.111 & 70.11 & & & & & \\
\hline f1LS & 67.05 & $\mathrm{O}$ & 68.21 & 64.16 & 69.94 & 69.94 & 69.94 & & & & & \\
\hline f2LS & 67.36 & 67.63 & 69.36 & $65 \cdot 32$ & 71.10 & 71.10 & 67.63 & & & & & \\
\hline foLSN & 66.67 & 66.09 & 67.24 & 70.69 & 70.69 & 70.69 & & & & & & \\
\hline f1LSN & 67.05 & 66.47 & 67.63 & 71.10 & 71.10 & 71.10 & & & & & & \\
\hline f2LSN & 67.05 & 67.05 & 68.21 & 71.68 & 71.68 & 71.68 & & & & & & \\
\hline Model9 & $\mathbf{1}$ & 2 & 3 & 4 & 5 & 6 & 7 & 8 & 9 & 10 & 11 & 12 \\
\hline foLS & 67.24 & 86.78 & O & 66.09 & 66.09 & 66.67 & 66.67 & 66.67 & 66.67 & & & \\
\hline f1LS & 67.05 & 86.71 & O & 65.90 & 65.90 & 66.47 & 66.47 & 66.47 & 66.47 & & & \\
\hline f2LS & 67.63 & 67.63 & 67.63 & 67.63 & 67.63 & 67.63 & 67.63 & & & & & \\
\hline foLSN & 66.67 & 66.09 & 67.24 & 70.69 & 70.69 & 68.97 & 68.97 & & & & & \\
\hline f1LSN & 67.05 & 66.47 & 67.63 & 71.10 & 71.10 & 70.52 & 69.94 & & & & & \\
\hline f2LSN & 67.63 & 67.63 & 68.79 & 72.25 & 72.25 & 70.52 & 68.79 & 69.94 & & & & \\
\hline Model1o & $\mathbf{1}$ & 2 & 3 & 4 & 5 & 6 & 7 & 8 & 9 & 10 & 11 & 12 \\
\hline foLS & 70.11 & 70.11 & 57.47 & 77.01 & 77.01 & 76.44 & 72.99 & & & & & \\
\hline f1LS & 69.94 & 69.94 & 57.23 & 76.88 & 76.88 & 76.30 & 72.83 & & & & & \\
\hline f2LS & 71.10 & 71.10 & 67.63 & 67.63 & 71.10 & 75.72 & 78.61 & 76.88 & & & & \\
\hline foLSN & 68.97 & $65.5^{2}$ & 66.09 & 66.09 & 67.24 & 64.94 & 71.84 & 72.99 & 69.54 & 68.39 & 72.41 & \\
\hline f1LSN & 69.94 & 66.47 & 66.47 & 66.47 & 67.63 & 66.47 & 72.83 & 73.99 & 70.52 & 69.36 & 73.99 & \\
\hline f2LSN & 71.10 & 67.63 & 67.63 & 65.90 & 68.79 & 66.47 & 73.99 & 73.41 & 72.83 & 72.25 & 70.52 & \\
\hline Model11 & $\mathbf{1}$ & 2 & 3 & 4 & 5 & 6 & 7 & 8 & 9 & 10 & 11 & 12 \\
\hline foLS & 70.11 & 70.11 & 70.11 & 70.11 & 70.11 & 70.11 & 66.09 & 64.94 & & & & \\
\hline f1LS & 69.94 & 69.94 & 69.94 & 69.94 & 69.94 & 69.94 & 65.90 & 67.40 & & & & \\
\hline f2LS & 71.10 & 87.86 & 87.86 & 100.00 & 60.12 & 73.99 & & & & & & \\
\hline foLSN & 69.54 & 66.09 & 64.94 & 64.37 & 66.09 & 63.79 & 72.99 & 73.56 & 74.71 & & & \\
\hline f1LSN & 69.54 & 66.47 & $65 \cdot 32$ & 64.74 & 66.47 & 65.32 & 74.57 & 74.57 & 76.30 & & & \\
\hline f2LSN & $70.5^{2}$ & 67.05 & 65.90 & 65.32 & 67.05 & 74.57 & 73.99 & 73.99 & 75.72 & & & \\
\hline Model12 & $\mathbf{1}$ & 2 & 3 & 4 & 5 & 6 & 7 & 8 & 9 & 10 & 11 & 12 \\
\hline foLS & 62.43 & 87.86 & 68.79 & 78.61 & 78.61 & 72.25 & 69.36 & 71.86 & 68.21 & 67.63 & & \\
\hline f1LS & 62.43 & 87.86 & 68.79 & 78.61 & 78.61 & 72.25 & 69.36 & 71.86 & 68.21 & & & \\
\hline f2LS & 67.63 & 87.86 & 78.03 & 78.61 & 78.61 & 76.72 & 75.14 & & & & & \\
\hline foLSN & 60.92 & 65.52 & 65.52 & 65.52 & 70.11 & 67.82 & 68.39 & & & & & \\
\hline f1LSN & 61.27 & 65.90 & 65.90 & 65.90 & $70.5^{2}$ & 69.36 & 69.36 & & & & & \\
\hline f2LSN & 67.63 & 67.63 & 67.63 & 68.21 & 67.05 & 67.63 & 67.63 & 67.05 & & & & \\
\hline Model13 & 1 & 2 & 3 & 4 & 5 & 6 & 7 & 8 & 9 & 10 & 11 & 12 \\
\hline foLS & 71.10 & 87.86 & O & 0 & 78.61 & 77.46 & 73.99 & 80.35 & 80.35 & 80.35 & 71.10 & \\
\hline f1LS & 71.10 & 87.86 & 0 & 0 & 78.61 & 77.46 & 73.99 & 80.35 & 80.35 & 80.35 & 71.10 & \\
\hline f2LS & 71.10 & 71.10 & 67.63 & 67.63 & 71.10 & 65.32 & 64.74 & 63.58 & 75.72 & 67.63 & 67.63 & 60.21 \\
\hline foLSN & 69.54 & 66.09 & 64.94 & 68.97 & 68.97 & 67.21 & 71.84 & 71.26 & 68.97 & 68.39 & 71.84 & \\
\hline f1LSN & 69.54 & 66.47 & 65.32 & 69.36 & 69.36 & 68.79 & 72.83 & 72.25 & 69.94 & 69.36 & 72.83 & \\
\hline f2LSN & 71.10 & 67.63 & 66.47 & 70.52 & $70.5^{2}$ & 73.99 & 73.99 & 73.99 & 72.25 & $70.5^{2}$ & 72.83 & \\
\hline
\end{tabular}


Table 10. Model Exponential Relations of the performing model

\begin{tabular}{|c|c|c|c|c|}
\hline Group Name & $\begin{array}{l}\text { Model } \\
\text { No }\end{array}$ & $\begin{array}{l}\text { Percent } \\
\text { Accuracy }\end{array}$ & $\begin{array}{l}\text { No of } \\
\text { Input }\end{array}$ & Model Exponential Relations of the performing model \\
\hline ModelfoLS & 6 & 77.59 & 7 & $0.005722\left(\frac{u^{*}}{v}\right)^{0.5}\left(\frac{v^{2}}{g y}\right)^{0.5}+-1.288 \mathrm{e}-005\left(\frac{R}{d_{50}}\right)\left(\frac{v^{2}}{g y}\right)+8.7177 \mathrm{e}-011 \frac{\left(\frac{R}{d_{50}}\right)^{1.5}}{\left(\frac{u^{*}}{v}\right)^{2}\left(\frac{v^{2}}{g y}\right)^{0.5}}$ \\
\hline Modelf1LS & 6 & 75.72 & 7 & $0.005722\left(\frac{u^{*}}{v}\right)^{0.5}\left(\frac{v^{2}}{g y}\right)^{0.5}+-1.288 \mathrm{e}-005\left(\frac{R}{d_{50}}\right)\left(\frac{v^{2}}{g y}\right)+8.7177 \mathrm{e}-011 \frac{\left(\frac{R}{d_{50}}\right)^{1.5}}{\left(\frac{u^{*}}{v}\right)^{2}\left(\frac{v^{2}}{g y}\right)^{0.5}}$ \\
\hline Modelf2LS & 9 & $75 \cdot 72$ & 7 & $\begin{array}{l}0.0047067\left(\frac{u^{*}}{v}\right)^{0.5}\left(\frac{v^{2}}{g y}\right)^{0.5}+-1.1407 \mathrm{e}-006\left(\frac{R}{d_{50}}\right)^{1.5}\left(\frac{v^{2}}{g y}\right)^{1.5}+6.7653 \mathrm{e}- \\
013 \frac{\left(\frac{R}{d_{50}}\right)^{1.5}\left(\frac{u^{*}}{\omega_{s}}\right)^{1.5}\left(\frac{v^{2}}{g y}\right)^{0.5}}{\left(\frac{u^{*}}{v}\right)^{2}\left(\frac{v s}{\omega}\right)^{1.5}}\end{array}$ \\
\hline ModelfoLSN & 11 & 75.29 & 7 & $0.13155 \frac{\left(\frac{u^{*}}{\omega_{s}}\right)^{0.5}\left(\frac{v^{2}}{g y}\right)}{\left(\frac{R}{d_{50}}\right)^{1.5}\left(\frac{u^{*}}{v}\right)^{2}}+7.7383 \frac{\left(\frac{u^{*}}{v}\right)^{2}\left(\frac{v^{2}}{g y}\right)}{\left(\frac{R}{d_{50}}\right)^{0.5}\left(\frac{B}{y}\right)^{0.5}}+2.1708 \mathrm{e}-007 \frac{\left(\frac{u^{*}}{\omega_{s}}\right)^{0.5}}{\left(\frac{u^{*}}{v}\right)^{1.5}\left(\frac{v^{2}}{g y}\right)^{1.5}}$ \\
\hline Modelf1LSN & 11 & 76.30 & 7 & $0.13155 \frac{\left(\frac{u^{*}}{\omega_{s}}\right)^{0.5}\left(\frac{v^{2}}{g y}\right)}{\left(\frac{R}{d_{50}}\right)^{1.5}\left(\frac{u^{*}}{v}\right)^{2}}+7.7383 \frac{\left(\frac{u^{*}}{v}\right)^{2}\left(\frac{v^{2}}{g y}\right)}{\left(\frac{R}{d_{50}}\right)^{0.5}\left(\frac{B}{y}\right)^{0.5}}+2.1708 \mathrm{e}-007 \frac{\left(\frac{u^{*}}{\omega_{s}}\right)^{0.5}}{\left(\frac{u^{*}}{v}\right)^{1.5}\left(\frac{v^{2}}{g y}\right)^{1.5}}$ \\
\hline Modelf2LSN & 10 & 76.88 & 7 & $8.9645 \frac{\left(\frac{v^{2}}{g y}\right)^{1.5}}{\left(\frac{R}{d_{50}}\right)\left(\frac{B}{y}\right)^{0.5}}\left(\frac{\omega}{u^{*}}\right)^{1.5}+1.4889 \frac{\left(\frac{u^{*}}{v}\right)^{2}\left(\frac{v^{2}}{g y}\right)^{0.5}}{\left(\frac{R}{d_{50}}\right)^{0.5}\left(\frac{B}{y}\right)^{0.5}}+2.0793 \mathrm{e}-007 \frac{\left(\frac{u^{*}}{\omega_{s}}\right)^{0.5}}{\left(\frac{u^{*}}{v}\right)^{1.5}\left(\frac{v^{2}}{g y}\right)^{1.5}}$ \\
\hline Model2foLS & 8 & 69.54 & 4 & $0.00096449\left(\frac{u^{*}}{v}\right)^{0.5}+0 \frac{\left(\frac{R}{d_{50}}\right)^{1.5}}{\left(\frac{B}{y}\right)\left(\frac{u^{*}}{\omega_{s}}\right)^{0.5}}+0 \frac{\left(\frac{R}{d_{50}}\right)^{2}\left(\frac{u^{*}}{\omega_{s}}\right)^{1.5}\left(\frac{v^{2}}{g y}\right)^{0.5}}{\left(\frac{B}{y}\right)^{2}\left(\frac{u^{*}}{\omega_{s}}\right)^{0.5}\left(\frac{u^{*}}{v}\right)}$ \\
\hline Model2f1LS & 8 & 69.94 & 4 & $0.00096449\left(\frac{u^{*}}{v}\right)^{0.5}+0 \frac{\left(\frac{R}{d_{50}}\right)^{1.5}}{\left(\frac{B}{y}\right)\left(\frac{u^{*}}{\omega_{s}}\right)^{0.5}}+0 \frac{\left(\frac{R}{d_{50}}\right)^{2}\left(\frac{u^{*}}{\omega_{s}}\right)^{1.5}\left(\frac{v^{2}}{g y}\right)^{0.5}}{\left(\frac{B}{y}\right)^{2}\left(\frac{u^{*}}{\omega_{s}}\right)^{0.5}\left(\frac{u^{*}}{v}\right)}$ \\
\hline $\begin{array}{l}\text { Model2f2LS } \\
* * *\end{array}$ & 6 & 100 & 4 & $\mathrm{O} \frac{1}{\left(\frac{R}{d_{50}}\right)^{2}}+0.015724 \frac{\left(\frac{u^{*}}{v}\right)^{0.5}}{\left(\frac{R}{d_{50}}\right)^{0.5}}+\mathrm{O} \frac{\left(\frac{R}{d_{50}}\right)^{2}}{\left(\frac{B}{y}\right)^{2}\left(\frac{u^{*}}{v}\right)^{2}}$ \\
\hline Model2foLSN & 1 & 69.54 & 4 & $0.00096449\left(\frac{u^{*}}{v}\right)^{0.5}$ \\
\hline Model2f1LSN & 1 & 69.94 & 4 & $0.00096449\left(\frac{u^{*}}{v}\right)^{0.5}$ \\
\hline Model2f2LSN & 1 & 71.10 & 4 & $0.00096449\left(\frac{u^{*}}{v}\right)^{0.5}$ \\
\hline Model3foLS & 6 & 98.28 & 5 & $\mathrm{O} \frac{1}{\left(\frac{R}{d_{50}}\right)^{2}}+0.015724 \frac{\left(\frac{u^{*}}{v}\right)^{0.5}}{\left(\frac{R}{d_{50}}\right)^{0.5}}+\mathrm{O} \frac{\left(\frac{R}{d_{50}}\right)^{2}}{\left(\frac{B}{y}\right)^{2}\left(\frac{u^{*}}{v}\right)^{2}}$ \\
\hline Model3f1LS & 6 & 98.27 & 5 & $\mathrm{O} \frac{1}{\left(\frac{R}{d_{50}}\right)^{2}}+0.015724 \frac{\left(\frac{u^{*}}{v}\right)^{0.5}}{\left(\frac{R}{d_{50}}\right)^{0.5}}+\mathrm{O} \frac{\left(\frac{R}{d_{50}}\right)^{2}}{\left(\frac{B}{y}\right)^{2}\left(\frac{u^{*}}{v}\right)^{2}}$ \\
\hline Model3f2LS & 1 & 71.10 & 5 & $0.00096449\left(\frac{u^{*}}{v}\right)^{0.5}$ \\
\hline Model3foLSN & 9 & 70.11 & 5 & $31.2116 \frac{\left(\frac{u^{*}}{\omega_{s}}\right)^{2}}{\left(\frac{R}{d_{50}}\right)^{2}\left(\frac{B}{y}\right)\left(\frac{u^{*}}{v}\right)^{2}}+0.00022426\left(\frac{B}{y}\right)^{0.5}\left(\frac{u^{*}}{v}\right)+1.4844 \mathrm{e}-007 \frac{\left(\frac{R}{d_{50}}\right)^{1.5}}{\left(\frac{B}{y}\right)^{2}\left(\frac{u^{*}}{v}\right)^{1.5}}$ \\
\hline Model3f1LSN & 9 & 71.86 & 5 & $31.2116 \frac{\left(\frac{u^{*}}{\omega_{s}}\right)^{2}}{\left(\frac{R}{d_{50}}\right)^{2}\left(\frac{B}{y}\right)\left(\frac{u^{*}}{v}\right)^{2}}+0.00022426\left(\frac{B}{y}\right)^{0.5}\left(\frac{u^{*}}{v}\right)+1.4844 \mathrm{e}-007 \frac{\left(\frac{R}{d_{50}}\right)^{1.5}}{\left(\frac{B}{y}\right)^{2}\left(\frac{u^{*}}{v}\right)^{1.5}}$ \\
\hline
\end{tabular}




\begin{tabular}{|c|c|c|c|c|}
\hline Model3f2LSN & 1 & 71.10 & 5 & $0.00096449\left(\frac{u^{*}}{v}\right)^{0.5}$ \\
\hline Model4foLS & 1 & 70.11 & 6 & $0.00096449\left(\frac{u^{*}}{v}\right)^{0.5}$ \\
\hline Model4fiLS & 1 & 69.94 & 6 & $0.00096449\left(\frac{u^{*}}{v}\right)^{0.5}$ \\
\hline Model4f2LS & 3 & 76.88 & 6 & $+-0.00011109 \frac{1}{\left(\frac{\omega}{u^{*}}\right)^{1.5}\left(\frac{u^{*}}{v}\right)^{1.5}}+0.0010153 \frac{1}{\left(\frac{\omega}{u^{*}}\right)^{0.5}}$ \\
\hline Model 4 foLSN & 9 & 74.71 & 6 & $18.7575 \frac{\left(\frac{v s}{\omega}\right)^{0.5}}{\left(\frac{R}{d_{50}}\right)\left(\frac{B}{y}\right)}+1.3436 \mathrm{e}-007 \frac{1}{\left(\frac{B}{y}\right)\left(\frac{\omega}{u^{*}}\right)^{2}\left(\frac{u^{*}}{v}\right)^{1.5}\left(\frac{v s}{\omega}\right)^{1.5}}+0.00018294\left(\frac{B}{y}\right)^{0.5}\left(\frac{u^{*}}{v}\right)$ \\
\hline Model4f1LSN & 9 & 76.30 & 6 & $18.7575 \frac{\left(\frac{v s}{\omega}\right)^{0.5}}{\left(\frac{R}{d_{50}}\right)\left(\frac{B}{y}\right)}+1.3436 \mathrm{e}-007 \frac{1}{\left(\frac{B}{y}\right)\left(\frac{\omega}{u^{*}}\right)^{2}\left(\frac{u^{*}}{v}\right)^{1.5}\left(\frac{v s}{\omega}\right)^{1.5}}+0.00018294\left(\frac{B}{y}\right)^{0.5}\left(\frac{u^{*}}{v}\right)$ \\
\hline Model 4 f2LSN & 9 & 76.30 & 6 & $18.7575 \frac{\left(\frac{v s}{\omega}\right)^{0.5}}{\left(\frac{R}{d_{50}}\right)\left(\frac{B}{y}\right)}+1.3436 \mathrm{e}-007 \frac{\left(\frac{u^{*}}{\omega_{s}}\right)^{2}}{\left(\frac{B}{y}\right)\left(\frac{u^{*}}{v}\right)^{1.5}\left(\frac{v s}{\omega}\right)^{1.5}}+0.00018294\left(\frac{B}{y}\right)^{0.5}\left(\frac{u^{*}}{v}\right)$ \\
\hline Model5foLS & 2 & 86.78 & 4 & $0.00041211\left(\frac{u^{*}}{\omega_{s}}\right)^{0.5}\left(\frac{\omega}{u^{*}}\right)^{0.5}$ \\
\hline Model5fiLS & - & - & - & - \\
\hline Model5f2LS & - & - & - & - \\
\hline Model5foLSN & 7 & 68.39 & 4 & $3936.6496 \frac{\left(\frac{u^{*}}{\omega_{s}}\right)^{2}}{\left(\frac{R}{d_{50}}\right)^{2}\left(\frac{B}{y}\right)^{1.5}}+0.0003356\left(\frac{u^{*}}{\omega_{s}}\right)^{0.5}\left(\frac{\omega}{u^{*}}\right)^{0.5}+1.5921 \mathrm{e}-005 \frac{\left(\frac{R}{d_{50}}\right)}{\left(\frac{B}{y}\right)^{2}}$ \\
\hline Model5f1LSN & 6 & 69.36 & 4 & 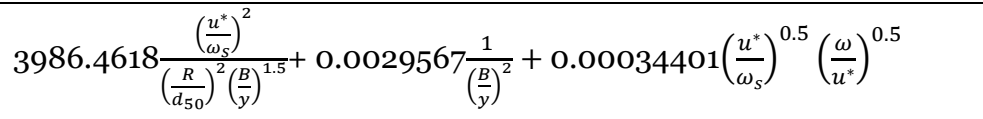 \\
\hline Model 5 f2LSN & 5 & 69.36 & 4 & $3986.4618 \frac{\left(\frac{u^{*}}{\omega_{s}}\right)^{2}}{\left(\frac{R}{d_{50}}\right)^{2}\left(\frac{B}{y}\right)^{1.5}}+0.0029567 \frac{1}{\left(\frac{B}{y}\right)^{2}}+0.00034401 \frac{1}{\left(\frac{B}{y}\right)}$ \\
\hline Model6foLS & 2 & 86.78 & 5 & $\mathrm{O} \frac{1}{\left(\frac{R}{d_{50}}\right)^{1.5}}+0.006834 \frac{1}{\left(\frac{R}{d_{50}}\right)^{0.5}}$ \\
\hline Model6f1LS & 2 & 86.71 & 5 & $\mathrm{O} \frac{1}{\left(\frac{R}{d_{50}}\right)^{1.5}}+0.006834 \frac{1}{\left(\frac{R}{d_{50}}\right)^{0.5}}$ \\
\hline Model6f2LS & 2 & 87.28 & 5 & $\mathrm{O} \frac{1}{\left(\frac{R}{d_{50}}\right)^{1.5}}+0.006834 \frac{1}{\left(\frac{R}{d_{50}}\right)^{0.5}}$ \\
\hline Model6foLSN & 5 & 70.11 & 5 & $0.31667 \frac{\left(\frac{v s}{\omega}\right)}{\left(\frac{R}{d_{50}}\right)^{0.5}\left(\frac{u^{*}}{\omega_{s}}\right)}+2.461 \mathrm{e}-007 \frac{1}{\left(\frac{v s}{\omega}\right)^{2}\left(\frac{v s}{\omega}\right)^{1.5}}$ \\
\hline Model6f1LSN & 5 & 70.52 & 5 & $0.31667 \frac{\left(\frac{v v}{\omega}\right)}{\left(\frac{R}{d_{50}}\right)^{0.5}\left(\frac{u^{*}}{\omega_{s}}\right)}+2.461 \mathrm{e}-007 \frac{1}{\left(\frac{v s}{\omega}\right)^{2}\left(\frac{v v}{\omega}\right)^{1.5}}$ \\
\hline Model6f2LSN & 5 & 71.68 & 5 & $0.31667 \frac{\left(\frac{\omega}{u^{*}}\right)\left(\frac{v s}{\omega}\right)}{\left(\frac{R}{d_{50}}\right)^{0.5}}+2.461 \mathrm{e}-007 \frac{\left(\frac{u^{*}}{\omega_{s}}\right)^{2}}{\left(\frac{v s}{\omega}\right)^{1.5}}$ \\
\hline Model7foLS & 5 & 70.11 & 6 & $\mathrm{o}\left(\frac{\omega}{u^{*}}\right)^{0.5}+0.0022795\left(\frac{R}{d_{50}}\right)^{0.5}\left(\frac{v^{2}}{g y}\right)^{0.5}+-2.622 e-005\left(\frac{R}{d_{50}}\right)\left(\frac{v^{2}}{g y}\right)$ \\
\hline Model7f1LS & 5 & 69.36 & 6 & $\mathrm{o}\left(\frac{\omega}{u^{*}}\right)^{0.5}+0.0022795\left(\frac{R}{d_{50}}\right)^{0.5}\left(\frac{v^{2}}{g y}\right)^{0.5}+-2.622 e-005\left(\frac{R}{d_{50}}\right)\left(\frac{v^{2}}{g y}\right)$ \\
\hline Model7f2LS & 3 & 69.36 & 6 & $3.36883 \mathrm{e}-005\left(\frac{R}{d_{50}}\right)^{0.5}+-4.3848 e-005\left(\frac{R}{d_{50}}\right)^{1.5}\left(\frac{v^{2}}{g y}\right)$ \\
\hline
\end{tabular}




\begin{tabular}{|c|c|c|c|c|}
\hline Model7foLSN & 4 & 72.99 & 6 & $20.2894 \frac{\left(\frac{v s}{\omega}\right)^{0.5}}{\left(\frac{R}{d_{50}}\right)\left(\frac{B}{y}\right)}+6.2504 \mathrm{e}-005 \frac{1}{\left(\frac{v g}{g y}\right)^{0.5}}$ \\
\hline Model7f1LSN & 4 & 73.41 & 6 & $20.2894 \frac{\left(\frac{v s}{\omega}\right)^{0.5}}{\left(\frac{R}{d_{50}}\right)\left(\frac{B}{y}\right)}+6.2504 \mathrm{e}-005 \frac{1}{\left(\frac{v g}{g y}\right)^{0.5}}$ \\
\hline Model7f2LSN & 8 & 76.30 & 6 & 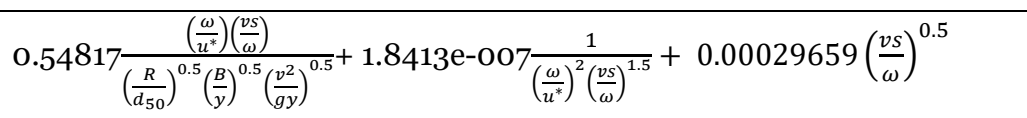 \\
\hline Model8foLS & 5 & 70.11 & 4 & 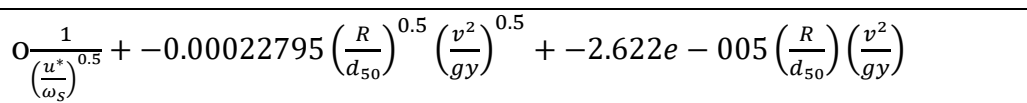 \\
\hline Model8f1LS & 5 & 69.94 & 4 & $\mathrm{o} \frac{1}{\left(\frac{u^{*}}{\omega_{s}}\right)^{0.5}}+-0.00022795\left(\frac{R}{d_{50}}\right)^{0.5}\left(\frac{v^{2}}{g y}\right)^{0.5}+-2.622 e-005\left(\frac{R}{d_{50}}\right)\left(\frac{v^{2}}{g y}\right)$ \\
\hline Model8f2LS & 5 & 71.10 & 4 & $\mathrm{o}\left(\frac{v^{2}}{g y}\right)+3.1645 e-005\left(\frac{R}{d_{50}}\right)^{0.5}+-9.0277 e-006\left(\frac{R}{d_{50}}\right)\left(\frac{v^{2}}{g y}\right)$ \\
\hline Model8foLSN & 4 & 70.69 & 4 & $8.3922 \frac{\left(\frac{v^{2}}{g y}\right)^{1.5}}{\left(\frac{R}{d_{50}}\right)\left(\frac{B}{y}\right)^{0.5}}+7.6601 \mathrm{e}-005 \frac{1}{\left(\frac{v^{2}}{g y}\right)^{0.5}}$ \\
\hline Model8fıLSN & 4 & 71.10 & 4 & $8.3922 \frac{\left(\frac{v v}{g y}\right)^{1.5}}{\left(\frac{R}{d_{50}}\right)\left(\frac{B}{y}\right)^{0.5}}+7.6601 \mathrm{e}-005 \frac{1}{\left(\frac{v}{g y}\right)^{0.5}}$ \\
\hline Model8f2LSN & 4 & 71.68 & 4 & $8.3922 \frac{\left(\frac{v^{2}}{g y}\right)^{1.5}}{\left(\frac{R}{d_{50}}\right)\left(\frac{B}{y}\right)^{0.5}}+7.6601 \mathrm{e}-005 \frac{1}{\left(\frac{v^{2}}{g y}\right)^{0.5}}$ \\
\hline ModelgfoLS & 2 & 86.78 & 5 & $\mathrm{O} \frac{1}{\left(\frac{R}{d_{50}}\right)^{1.5}}+0.0068341 \frac{1}{\left(\frac{R}{d_{50}}\right)^{0.5}}$ \\
\hline ModelgfiLS & 2 & 86.71 & 5 & $0 \frac{1}{\left(\frac{R}{d_{50}}\right)^{1.5}}+0.0068341 \frac{1}{\left(\frac{R}{d_{50}}\right)^{0.5}}$ \\
\hline Modelgf2LS & 1 & 67.63 & 5 & $0.00041211\left(\frac{B}{y}\right)^{0.5}$ \\
\hline ModelgfoLSN & 4 & 70.69 & 5 & $8.3922 \frac{\left(\frac{v^{2}}{g y}\right)^{1.5}}{\left(\frac{R}{d_{50}}\right)\left(\frac{(B)}{y}\right)^{0.5}}+7.6601 \mathrm{e}-005 \frac{1}{\left(\frac{v^{2}}{g y}\right)^{0.5}}$ \\
\hline ModelgfiLSN & 4 & 71.10 & 5 & $8.3922 \frac{\left(\frac{v^{2}}{g y}\right)^{1.5}}{\left(\frac{R}{d_{50}}\right)\left(\frac{B}{y}\right)^{0.5}}+7.6601 \mathrm{e}-005 \frac{1}{\left(\frac{v^{2}}{g y}\right)^{0.5}}$ \\
\hline Modelgf2LSN & 4 & 72.25 & 5 & $8.3922 \frac{\left(\frac{v^{2}}{g y}\right)^{1.5}}{\left(\frac{R}{d_{50}}\right)\left(\frac{B}{y}\right)^{0.5}}+7.6601 \mathrm{e}-005 \frac{1}{\left(\frac{v^{2}}{g y}\right)^{0.5}}$ \\
\hline Model1ofoLS & 4 & 77.01 & 6 & 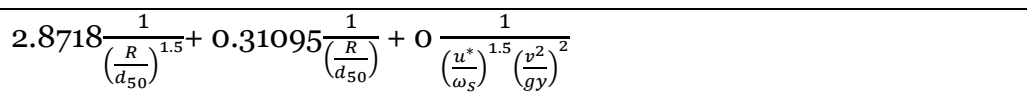 \\
\hline Model1of1LS & 4 & 76.88 & 6 & $2.8718 \frac{1}{\left(\frac{R}{d_{50}}\right)^{1.5}}+0.31095 \frac{1}{\left(\frac{R}{d_{50}}\right)}+0 \frac{1}{\left(\frac{u^{*}}{\omega_{s}}\right)^{1.5}\left(\frac{v^{2}}{g y}\right)^{2}}$ \\
\hline Model1of2LS & 7 & 78.61 & 6 & $\begin{array}{l}0.005722\left(\frac{u^{u}}{v}\right)^{0.5}\left(\frac{v^{2}}{g y}\right)^{0.5}+-1.288 e-005\left(\frac{R}{d_{50}}\right)\left(\frac{v^{2}}{g y}\right)+8.7177 e- \\
011 \frac{\left(\frac{R}{d s_{0}}\right)^{1.5}}{\left(\frac{u^{*}}{v}\right)^{2}\left(\frac{v^{2}}{g y}\right)^{0.5}}\end{array}$ \\
\hline Model1ofoLSN & 8 & 72.99 & 6 & $0.10676 \frac{\left(\frac{u^{*}}{v}\right)\left(\frac{v^{2}}{g y}\right)^{0.5}}{\left(\frac{R}{d_{50}}\right)^{0.5}}+3.9855 \mathrm{e}-007 \frac{\left(\frac{u^{*}}{\omega_{s}}\right)}{\left(\frac{B}{y}\right)^{0.5}\left(\frac{u^{*}}{v}\right)^{2}\left(\frac{v^{2}}{g y}\right)^{1.5}}+0.00016018\left(\frac{u^{*}}{v}\right)^{1.5}$ \\
\hline
\end{tabular}




\begin{tabular}{|c|c|c|c|c|}
\hline Model1of1LSN & 8 & 73.99 & 6 & $0.10676 \frac{\left(\frac{u^{*}}{v}\right)\left(\frac{v^{2}}{g y}\right)^{0.5}}{\left(\frac{R}{d_{50}}\right)^{0.5}}+3.9855 \mathrm{e}-007 \frac{\left(\frac{u^{*}}{\omega_{s}}\right)}{\left(\frac{B}{y}\right)^{0.5}\left(\frac{u^{*}}{v}\right)^{2}\left(\frac{v^{2}}{g y}\right)^{1.5}}+0.00016018\left(\frac{u^{*}}{v}\right)^{1.5}$ \\
\hline Model1of2LSN & 7 & 73.99 & 6 & $4.6159 \frac{\left(\frac{u^{*}}{v}\right)^{1.5}\left(\frac{v^{2}}{g y}\right)}{\left(\frac{R}{d_{50}}\right)^{0.5}\left(\frac{B}{y}\right)^{0.5}}+2.1553 \mathrm{e}-007 \frac{\left(\frac{u^{*}}{\omega_{s}}\right)^{0.5}}{\left(\frac{u^{*}}{v}\right)^{1.5}\left(\frac{v^{2}}{g y}\right)^{1.5}}$ \\
\hline Model11foLS & 1 & 70.11 & 5 & $0.00096449\left(\frac{u^{*}}{v}\right)^{0.5}$ \\
\hline Model11f1LS & 1 & 69.94 & 5 & $0.00096449\left(\frac{u^{*}}{v}\right)^{0.5}$ \\
\hline $\begin{array}{l}\text { Model11f2LS } \\
* * *\end{array}$ & 4 & 100.00 & 5 & $\mathrm{O} \frac{1}{\left(\frac{R}{d_{50}}\right)^{2}}+0.015724 \frac{\left(\frac{u^{*}}{v}\right)^{0.5}}{\left(\frac{R}{d_{50}}\right)^{0.5}}+\mathrm{O} \frac{1}{\left(\frac{u^{*}}{v}\right)^{1.5}}$ \\
\hline Model11foLSN & 9 & 74.71 & 5 & $18.7575 \frac{\left(\frac{v s}{\omega}\right)^{0.5}}{\left(\frac{R}{s_{50}}\right)\left(\frac{B}{y}\right)}+1.3436 \mathrm{e}-007 \frac{\left(\frac{u^{*}}{\omega_{s}}\right)^{2}}{\left(\frac{B}{y}\right)\left(\frac{u^{*}}{v}\right)^{1.5}\left(\frac{v s}{\omega}\right)^{1.5}}+0.00018294\left(\frac{B}{y}\right)^{0.5}\left(\frac{u^{*}}{v}\right)$ \\
\hline Model11f1LSN & 9 & 76.30 & 5 & $18.7575 \frac{\left(\frac{v s}{\omega}\right)^{0.5}}{\left(\frac{R}{d_{50}}\right)\left(\frac{B}{y}\right)}+1.3436 \mathrm{e}-007 \frac{\left(\frac{u^{*}}{\omega_{s}}\right)^{2}}{\left(\frac{B}{y}\right)\left(\frac{u^{*}}{v}\right)^{1.5}\left(\frac{v s}{\omega}\right)^{1.5}}+0.00018294\left(\frac{B}{y}\right)^{0.5}\left(\frac{u^{*}}{v}\right)$ \\
\hline Model11f2LSN & 6 & 74.57 & 5 & $20.6487 \frac{\left(\frac{v s}{\omega}\right)^{0.5}}{\left(\frac{R}{d_{50}}\right)\left(\frac{B}{y}\right)}+0.00078901\left(\frac{u^{*}}{v}\right)+1.4956 \mathrm{e}-007 \frac{\left(\frac{u^{*}}{\omega_{s}}\right)^{2}}{\left(\frac{v s}{\omega}\right)^{1.5}}$ \\
\hline Model12foLS & 2 & 87.86 & 4 & $\mathrm{O} \frac{1}{\left(\frac{R}{d_{50}}\right)^{1.5}}+0.0068341 \frac{1}{\left(\frac{R}{d_{50}}\right)^{0.5}}$ \\
\hline Model12f1LS & 2 & 87.86 & 4 & $\mathrm{O} \frac{1}{\left(\frac{R}{d_{50}}\right)^{1.5}}+0.0068341 \frac{1}{\left(\frac{R}{d_{50}}\right)^{0.5}}$ \\
\hline Model12f2LS & 2 & 87.86 & 4 & $\mathrm{O} \frac{1}{\left(\frac{R}{d_{50}}\right)^{1.5}}+0.0068341 \frac{1}{\left(\frac{R}{d_{50}}\right)^{0.5}}$ \\
\hline Model12foLSN & 5 & 70.11 & 4 & $0.31667 \frac{\left(\frac{v s}{\omega}\right)}{\left(\frac{R}{d_{50}}\right)^{0.5}\left(\frac{u^{*}}{\omega_{s}}\right)}+2.461 \mathrm{e}-007 \frac{\left(\frac{u^{*}}{\omega_{s}}\right)^{2}}{\left(\frac{v s}{\omega}\right)^{1.5}}$ \\
\hline Model12f1LSN & 5 & 70.52 & 4 & $0.31667 \frac{\left(\frac{v s}{\omega}\right)}{\left(\frac{R}{d_{50}}\right)^{0.5}\left(\frac{u^{*}}{\omega_{s}}\right)}+2.461 \mathrm{e}-007 \frac{\left(\frac{u^{*}}{\omega_{s}}\right)^{2}}{\left(\frac{v s}{\omega}\right)^{1.5}}$ \\
\hline Model12f2LSN & 4 & 68.21 & 4 & $0.12605 \frac{\left(\frac{v s}{\omega}\right)^{1.5}}{\left(\frac{u^{*}}{\omega_{s}}\right)^{1.5}}+2.7393 \mathrm{e}-007 \frac{\left(\frac{u^{*}}{\omega_{s}}\right)^{2}}{\left(\frac{v s}{\omega}\right)^{1.5}}$ \\
\hline Model13foLS & 2 & 87.86 & 6 & $\mathrm{O} \frac{1}{\left(\frac{R}{d_{50}}\right)^{1.5}}+0.0068341 \frac{1}{\left(\frac{R}{d_{50}}\right)^{0.5}}$ \\
\hline Model13f1LS & 2 & 87.86 & 6 & $\mathrm{O} \frac{1}{\left(\frac{R}{d_{50}}\right)^{1.5}}+0.0068341 \frac{1}{\left(\frac{R}{d_{50}}\right)^{0.5}}$ \\
\hline Model13f2LS & 9 & 75.72 & 6 & $\begin{array}{l}0.00471\left(\frac{u^{*}}{v}\right)^{0.5}\left(\frac{v^{2}}{g y}\right)^{0.5}+-1.1512 e-006\left(\frac{R}{d_{50}}\right)^{1.5}\left(\frac{v^{2}}{g y}\right)^{1.5}+7.157 e- \\
013 \frac{\left(\frac{R}{d_{50}}\right)^{1.5}\left(\frac{u^{*}}{\omega_{s}}\right)\left(\frac{v^{2}}{g y}\right)^{2}}{\left(\frac{u^{*}}{v}\right)^{1 .}\left(\frac{v s}{\omega}\right)^{2}}\end{array}$ \\
\hline Model13foLSN & 7 & 71.84 & 6 & $15.2937 \frac{\left(\frac{v^{2}}{g y}\right)}{\left(\frac{R}{d_{50}}\right)\left(\frac{B}{y}\right)}+0.0077026\left(\frac{u^{*}}{v}\right)^{1.5}\left(\frac{v^{2}}{g y}\right)^{0.5}+2.0303 \mathrm{e}-007 \frac{\left(\frac{u^{*}}{\omega_{s}}\right)^{2}}{\left(\frac{v s}{\omega}\right)^{1.5}}$ \\
\hline Model13f1LSN & 7 & 72.83 & 6 & $15.2937 \frac{\left(\frac{v^{2}}{g y}\right)}{\left(\frac{R}{d_{50}}\right)\left(\frac{B}{y}\right)}+0.0077026\left(\frac{u^{*}}{v}\right)^{1.5}\left(\frac{v^{2}}{g y}\right)^{0.5}+2.0303 \mathrm{e}-007 \frac{\left(\frac{u^{*}}{\omega_{s}}\right)^{2}}{\left(\frac{v s}{\omega}\right)^{1.5}}$ \\
\hline
\end{tabular}




\begin{tabular}{|l|l|l|l|l|}
\hline Model13f2LSN & 6 & 73.99 & 6 & $4.5415 \frac{\left(\frac{u^{*}}{v}\right)^{1.5}\left(\frac{v^{2}}{g y}\right)}{\left(\frac{R}{d_{50}}\right)^{0.5}\left(\frac{B}{y}\right)^{0.5}}+2.4772 \mathrm{e}-007 \frac{\left(\frac{u^{*}}{\omega_{s}}\right)^{2}}{\left(\frac{v s}{\omega}\right)^{1.5}}$ \\
\hline
\end{tabular}

Note: ${ }^{* *}$ Best model 
Figure 1 shows performance of Group Model2f2LS (Model 1 to predict the measured values within an acceptable limit. until Model 7). Seven newly developed models have shown The model that best predicts the measured values is Model 6.

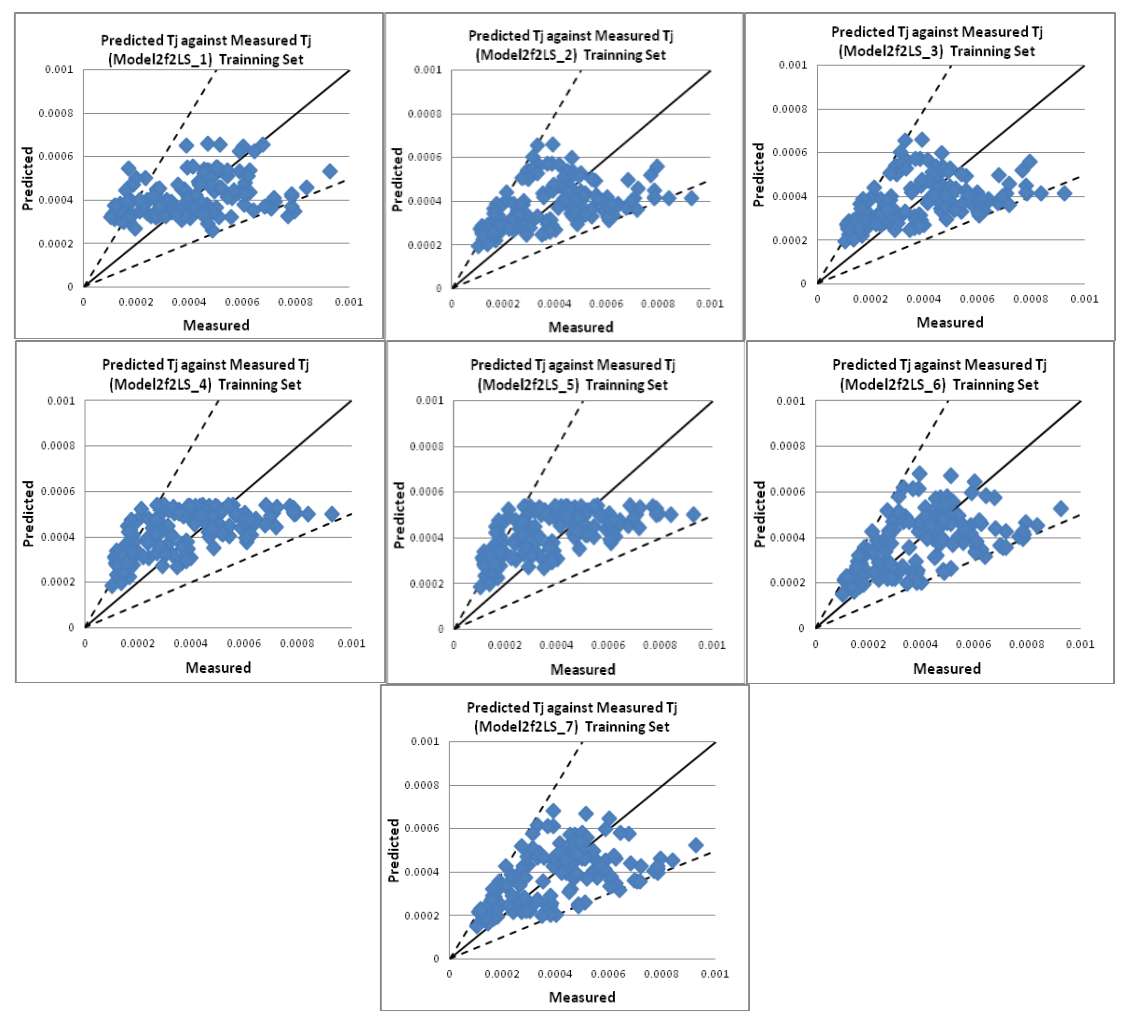

Figure 1. Model performance for Group Model2f2LS (Model 1 until Model 7)

Models in Figure 2 exhibit similar trends to Figure 1. Analyses suggest that Model 4 exhibit the best predictions compare to other 5 models.

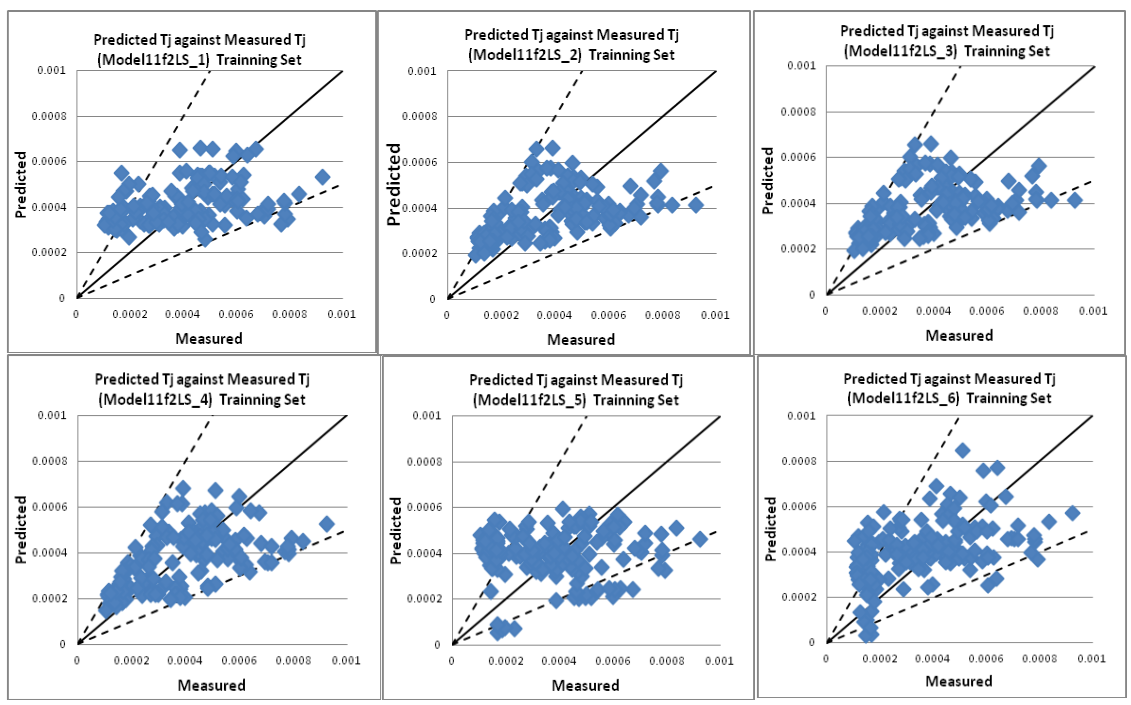

Figure 2. Model performance Group Model11f2LS (Model 1 until Model 6)

Results with data confirmation from Table 10 suggest that

(Model2f2LS) and Model 4 (Model 11f2LS)are:

Model 6 (Model2f2LS) yield 100 percent accuracy in prediction. The exponential relations of Model 6 
Model 6 (Model2f2LS)

$$
\frac{Q t}{Q}=0 \frac{1}{\left(\frac{R}{d_{50}}\right)^{2}}+0.015724 \frac{\left(\frac{u^{*}}{v}\right)^{0.5}}{\left(\frac{R}{d_{50}}\right)^{0.5}}+0 \frac{\left(\frac{R}{d_{50}}\right)^{2}}{\left(\frac{B}{y}\right)^{2}\left(\frac{u^{*}}{v}\right)^{2}}
$$

Model 4 (Model 11f2LS)

$$
\frac{Q t}{Q}=0 \frac{1}{\left(\frac{R}{d_{50}}\right)^{2}}+0.015724 \frac{\left(\frac{u^{*}}{v}\right)^{0.5}}{\left(\frac{R}{d_{50}}\right)^{0.5}}+0 \frac{1}{\left(\frac{u^{*}}{v}\right)^{1.5}}
$$

Both models 6 and 4 (Equations 1 and 2) suggest $\frac{U^{*}}{V}$ (ratio of shear velocity to flow velocity) and $\frac{R}{d_{50}}$ (ratio of hydraulic radius to mean sediment diameter) as the most significant and influential parameters. With the above discovery, the general expression for sediment concentration can be expressed as follows;

$$
\frac{Q t}{Q}=0.015724 \frac{\left(U^{*} / V\right)^{0.5}}{\left(R / d_{50}\right)^{0.5}}
$$

where; $Q t$ is sediment total load $(\mathrm{kg} / \mathrm{s}) ; V$ is flow velocity $(\mathrm{m} / \mathrm{s}), d_{50}$ is median diameter of sediment load $(\mathrm{m}), Q$ is flow discharge $(\mathrm{kg} / \mathrm{s}), U^{*}$ is shear velocity $(\mathrm{m} / \mathrm{s})$ and $R$ is hydraulic radius (m).

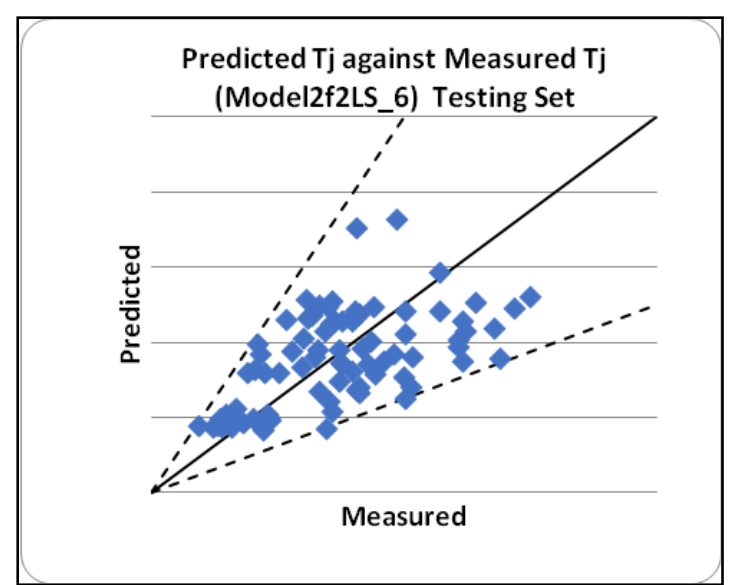

Figure 3. Graph of measured sediment total load versus predicted sediment total load using 82 testing data

\section{CONCLUSION}

Analyses carried out on the model parameters have indicated that two variables namely $\frac{U^{*}}{V}$ (ratio of shear velocity to flow velocity) and $\frac{R}{d_{50}}$ (ratio of hydraulic radius to mean sediment diameter) to be the most significant and influential parameters. The above is confirmed by the performance of the model with 100 percent prediction accuracy. This new model is an improved model of Ariffin (2004), of which the latter has used four model parameters as predictors. In the improved model, analyses have confirmed that only two parameters could predict with greater accuracy the measured sediment load values.

\section{ACKNOWLEDGEMENT}

The authors acknowledge the Department of Irrigation and Drainage (DID) of the Ministry of Natural Resources \& Environment, Malaysia, for their assistance in providing the data used in this study. 


\section{REFERENCES}

[1] Ackers, P \& White, WR 1973, 'Sediment transport: New approach and analysis', Journal of the Hydraulics Division, vol. 99, no. 11, pp. 20412060.

[2] Ariffin, J 2004, 'Development of sediment transport models for rivers in Malaysia using regression analysis and artificial neural networks', PhD thesis, Universiti Sains Malaysia, Penang, Malaysia.

[3] Ariffin, J 2017, Fluvial geomorphology anthropogenic agents of change and their implications, Penerbit Press Universiti Teknologi MARA, Malaysia.

[4] Azamathulla, HM, Ab Ghani, A, Zakaria, NA \& Chang, CK, Abu Hasan, Z 2010, 'Genetic programming approach to predict sediment concentration for Malaysia rivers', International Journal of Ecological Economics and Statistics, vol. 16 , no. 10 , pp. 53-64.

[5] Chang, CK, Ab Ghani, A, Zakaria, NA, Abu Hasan, Z \& Abdullah, R 2005, 'Sediment transport equation assessment for selected rivers in Malaysia', International Journal of River Basin Management, vol. 3, no. 3, pp. 203-208.

[6] Department of Irrigation and Drainage 2009, River sand mining management guideline, Department of Irrigation and Drainage, Kuala Lumpur.

[7] Department of Irrigation and Drainage 2013, Kajian pengumpulan data dan analisis Endapan Sungai, Final report vol. 1, pp. 49-56.

[8] Doglioni, A \& Simeone, V 2017, 'Evolutionary modelling of response of water table to precipitations', Journal of Hydrologic Engineering, vol. 22, no. 2, pp. 04016055.

[9] Draper, NR \& Smith, H 1998, Applied regression analysis, John Wiley and Sons, New York.

[10] Engelund, F \& Hansen, E 1967, A monograph on sediment transport in alluvial streams, Teknisk Forlag, Copenhagen.
[11] Ghorbani, A \& Hasanzadehshooiili, H 2018, 'Prediction of UCS and CBR of microsilica-lime stabilized sulphate silty sand using ANN and EPR Models; application to the deep soil mixing', Journal Soils and Foundations, vol. 58, no. 1, pp. 34-49.

[12] Giustolisi, O \& Savic, DA 2006, 'A symbolic data driven technique based on evolutionary polynomial regression', Journal of Hydroinformatics, vol. 8, no. 3, pp. 207-222.

[13] Giustolisi, O, Doglioni, A, Savic, DA \& di Pierro, F 2008, 'An evolutionary multiobjective strategy for the effective management of groundwater resources', Water Resources Research Journal, vol. 44, no. 1 , pp. 1-14

[14] Graf, WH \& Acaroglu, ER 1968, 'Sediment transport in conveyances systems, Part 1', International Association of Scientific Hydrology, vol. 13 , no. 2 , pp. $20-39$.

[15] Ibrahim, NA 2012, 'Penilaian dan Pembangunan Persamaan Pengangkutan Endapan Sungai-Sungai di Malaysia', Final thesis, Universiti Sains Malaysia, Penang, Malaysia.

[16] Karim, F 1998, 'Bed Material discharge prediction for non-uniform bed sediments', Hydraulic Engineering, vol. 124, no. 6, 597-604.

[17] Koza, JR 1992, Genetic programming: On the programming of computers by means of natural selection, A Bradford Book, The MIT Press, Massachusetts, London, England.

[18] Laucelli, D, Berardi, L \& Dogliono, A 2009, Evolutionary Polynomial Regression (EPR) Toolbox, Version 2.oSA (Stand Alone Version), Department of Civil and Environmental Engineering, Technical University of Bari, Italy.

[19] Molinas, A \& Wu, B 2001, 'Transport of Sediment in Large Sand-bed Rivers', Journal of Hydraulic Research, vol. 39, no. 2, pp. 135-146.

[20] Saleh, A 2016, 'Optimal sand removal capacity for in-stream mining', Master thesis, Universiti Sains 
Malaysia, Penang, Malaysia.

[21] Saleh, A, Abustan, I, Mohd Remy Rozainy, MAZ \& Sabtu, N 2017, 'Assessment of total bed material equations on selected Malaysia rivers', in AIP Conference Proceeding, vol. 1892, no. 1, pp. 070002.

[22] Savic, DA, Giutolisi, O, Berardi, L, Shepherd, W, Djordjevic, S \& Saul, A 2006, 'Modelling sewer failure by evolutionary computing', in Proceeding of the Institution of Civil Engineers, Water Management, vol. 159, no. 2, pp. 111-118.

[23] Shahin, MA, Maier, HR \& Jaksa, MB 2004, 'data division for developing neural networks applied to geotechnical engineering', Journal of Computing in Civil Engineering, ASCE, vol. 18, no. 2, pp. 105114.

[24] Sinnakaudan, SK, Ab Ghani, A, Ahmad, MS \& Zakaria, NA 2006, 'Multiple linear regression model for total bed material load prediction', Journal of Hydraulic Engineering, vol. 132, no. 5, pp. 521-528.

[25] Sulaiman, MS 2009, 'Sediment transport equation development for highland rivers in Malaysia', Master's thesis, Universiti Teknologi MARA, Malaysia.

[26] Yang, C.T 1973, 'Incipient Motion and Sediment Transport', Journal of the Hydraulics Division, vol. 99, no. 10, pp. 1679-1704.

[27] Yin, ZY, Jin, YF, Huang, HW \& Shen, SL 2016, 'Evolutionary polynomial regression-based model of clay compressibility using an enhanced hybrid real-coded genetic algorithm', Engineering Geology Journal, vol. 210, pp. 158-167. 